This item is the archived peer-reviewed author-version of:

Indirect 3D printed electrode mixers

\title{
Reference:
}

Hereijgers Jonas, Schalck Jonathan, Lölsberg Jonas, Wessling Matthias, Breugelmans Tom.- Indirect 3D printed electrode mixers ChemElectroChem - ISSN 2196-0216 - 6:2(2019), p. 378-382

Full text (Publisher's DOI): https://doi.org/10.1002/CELC.201801436

To cite this reference: https://hdl.handle.net/10067/1543890151162165141 


\section{Indirect 3D printed electrode mixers}

\begin{abstract}
Jonas Hereijgers ${ }^{\star[a]}$, Jonathan Schalck ${ }^{[a]}$, Jonas Lölsb Abstract: Electrode mixers are ordered 3D flow-through structures
with mixing properties that at the same time act as electrode. Because of their mixing properties, these structures enhance mass transfer at the electrode surface, increasing the limiting-current plateau. Typically operating at this plateau, the productivity of electrochemical reactors is limited by the mass transport of the reagents. This work presents the fabrication of all-metal electrode mixers through an indirect 3D printing method. Whereas other techniques suffer from design limitations or come with high capital cost, the proposed rapid prototyping method overcomes these limitations. Using this method, a helical and cubic electrode mixer were fabricated and their mass transfer properties were characterized and compared to a flat electrode by the limiting current method. Increasing the mass transfer up to $47 \%$ the standard flat electrode is outperformed, demonstrating the valorization potential of electrode mixers and the indirect 3D printing method.
\end{abstract}

\section{Introduction}

Typically, electrochemical reactors operate in the transportlimited regime ${ }^{[1]}$. In this regime the productivity is independent of the potential as the current is at its maximum (limiting current plateau). Only by shifting this limiting current plateau to higher values can the productivity be increased. This can be achieved by increasing mass transport towards the electrode ${ }^{[2-4]}$. To this end, electrode mixers were recently reported ${ }^{[5,6]}$. Electrode mixers are 3D shaped conductive structures that enhance mass transport towards the electrode by reducing concentration polarization at the electrode due to its mixing properties. As a result, the electrochemical community is more and more looking at $3 D$ electrodes to enhance the mass transfer in electrochemical processes, such as batteries ${ }^{[7-11]}$ or redox flow batteries ${ }^{[12,13]}$. However, fabrication of such structures at reasonable cost without a complicated synthesis protocol avoiding expensive machines is to date still a pressing problem especially when targeting all-metal structures. This inhibits experimental testing in a fast prototyping way. In reported work ${ }^{[5,6]}$, helical electrode mixers were constructed by selective laser melting (SLM). Downside of SLM is the challenging implementation of multi-materials ${ }^{[14]}$, not to mention the associated cost that comes with SLM (e.g. €500 for a single prototype ${ }^{[6]}$ ). Linkhorst et al. ${ }^{[14]}$ tackled these problems

[a] Dr. J. Hereijgers, J. Schalck, Prof. Dr. T. Breugelmans Advanced Reactor Technology

University of Antwerp

Universiteitsplein 1, 2610 Wilrjik, Belgium

E-mail: Jonas.Hereijgers@uantwerp.be

[b] J. Lölsberg, Prof. Dr. M. Wessling

Aachener Verfahrenstechnik-Chemical Process Engineering

RWTH Aachen University

Forckenbeckstr. 51, 52074 Aachen, Germany

[c] J. Lölsberg, Prof. Dr. M. Wessling

DWI - Leibniz Institute for Interactive Materials

Forckenbeckstr. 51, 52074 Aachen, Germany by demonstrating the concept of laserless additive manufacturing for electrode assemblies. Using additive manufacturing technology, 3D shaped electrodes were printed using a paste of metallic particles and cellulose as binder. Subsequently, this unsintered or green part was placed in an oven and sintered together. While the cellulose gave mechanical strength to the green part, the freedom of design was limited by the achievable aspect ratio (height/width). At large aspect ratios, the green part collapsed. Consequently, to the best of our knowledge there is to date no fabrication process being described that truly allows to design and fabricate all-metal electrode mixers free of such fabrication problems, which is robust, easy to implement and cheap. Here we demonstrate for the first time such a method to fabricate electrode mixers by indirect 3D printing.

\section{Materials and Methods}

\subsection{Chemicals}

Nickel powder $(<50 \mu \mathrm{m}, 99.7 \%)$ was purchased from SigmaAldrich (Belgium) and epoxy (Specifix 40) from Struers (The Netherlands). Potassium hexacyanoferrate(II) $\left(\mathrm{K}_{4} \mathrm{Fe}(\mathrm{CN})_{6} \cdot 3 \mathrm{H}_{2} \mathrm{O}\right)$, potassium hexacyanoferrate(III) $\left.\left(\mathrm{K}_{3} \mathrm{Fe}(\mathrm{CN})_{6}\right)\right)$, glycerol, toluene and sodium sulfate were purchased from Acros Organics (Belgium). Water was prepared in the laboratory (Milli-Q gradient, Millipore, USA). LimoSolve polymer was purchased from Formfutura. All used gases were purchased from Praxair.

\subsection{Indirect 3D printing}

Using Inventor (Autodesk) a mold of the electrode was designed. Next, this mold was printed in LimoSolve with a fused deposition molding 3D-printer (Ultimaker 3). LimoSolve was chosen over PVA despite its water solubility as LimoSolve less easily burned during printing, causing malfunction and was less prone to stringing, improving the printing quality and resolution. Subsequently, the mold was filled with a paste consisting of nickel powder (61.2 wt\%), epoxy (14.3 wt\%) and glycerol (24.5 wt\%) (Fig. 1). As case study nickel was used as it is a frequently applied electrode material for alkaline water electrolysis, a major electrochemical application ${ }^{[15-17]}$. Epoxy was mixed into the paste to give the green part mechanical stability. In protocols reported in literature such as the non-solvent method a polymer such as polyetherimide is used as binder ${ }^{[18]}$. This strategy, however, was not feasible as the mold blocks any solvent extraction, not to mention the freedom of design restriction to $2.5 \mathrm{D}$ designs (e.g. hollow fibers). In the non-solvent method, the polymer is first dissolved in a suited solvent, yielding a flexible paste. Next the paste is shaped (e.g. extrusion) and subsequently the polymer solidified by extracting only the solvent with a second solvent. To have a binder that could solidify inside the mold, epoxy was used as it chemically solidifies, omitting the need for a solvent extraction step. 


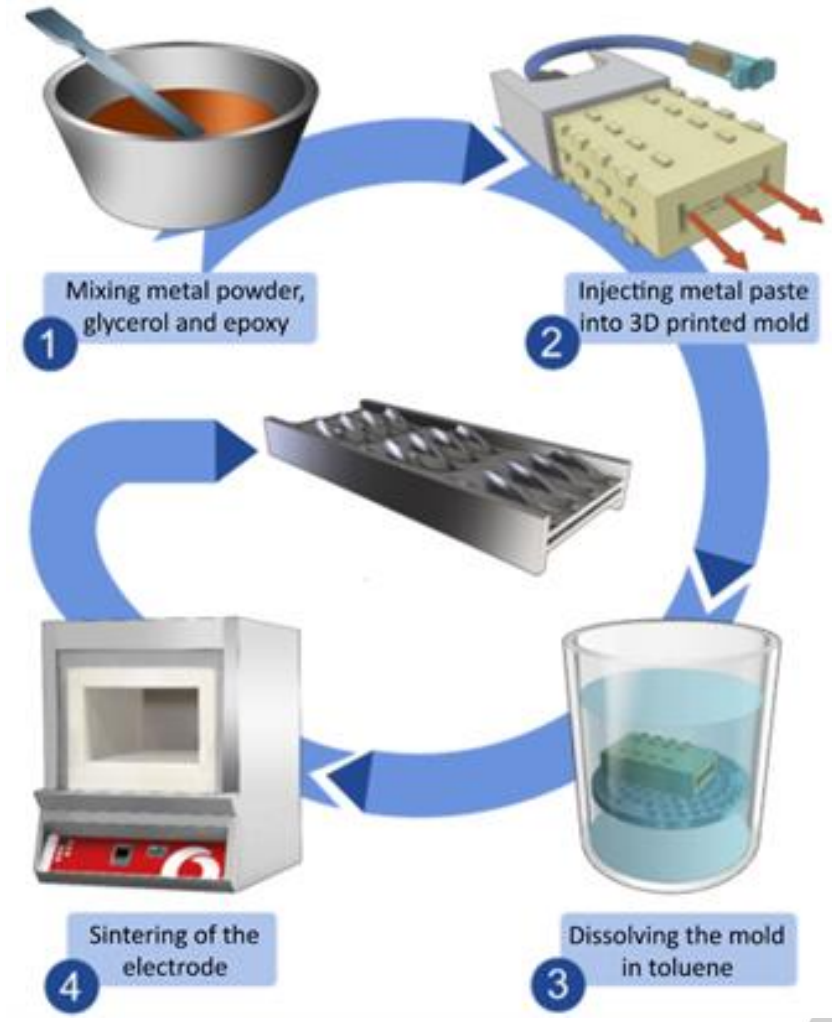

Figure 1. Indirect 3D printing method

After filling the mold, epoxy was cured for $3 \mathrm{~h}$ at $40^{\circ} \mathrm{C}$. Once the paste was solidified, the mold was removed by dissolving it in toluene, yielding the green part. Toluene was opted over limonene or acetone as toluene removed all LimoSolve within 48 hours and did not affect the epoxy and the mechanical stability of the green part. Next, this green part was sintered in two steps in a Carbolite oven (CWF 13/23 A.105). In the first step air was fed (50 mL $\min ^{-1}$ ) into the oven and the green part was gradually heated up. First the temperature was rapidly raised to $350^{\circ} \mathrm{C}$ at a rate of $5^{\circ} \mathrm{C}$ $\mathrm{min}^{-1}$ to reduce time. At $350^{\circ} \mathrm{C}$, the temperature was raised slowly to $600^{\circ} \mathrm{C}\left(1^{\circ} \mathrm{C} \mathrm{min}{ }^{-1}\right)$ to avoid stress in the green part. After 1 hour at $600^{\circ} \mathrm{C}$, to remove all binder, the temperature was increased to $1000^{\circ} \mathrm{C}\left(1^{\circ} \mathrm{C} \mathrm{min}^{-1}\right)$ followed by the same dwell time to sinter the metal particles. Finally, the samples were cooled down to room temperature at a rate of $1^{\circ} \mathrm{C} \mathrm{min}^{-1}$. In the second step, the gas feed was switched to a mixture of $5 \% \mathrm{H}_{2}$ in argon. To remove all air, the oven was flushed $(20 \mathrm{~L} \mathrm{~min}-1,15 \mathrm{~min})$ at ambient temperatures. After $15 \mathrm{~min}$ the flow rate was lowered to $60 \mathrm{~mL}$ $\mathrm{min}^{-1}$ and the temperature raised to $600^{\circ} \mathrm{C}\left(1^{\circ} \mathrm{C} \mathrm{min}^{-1}\right)$, followed by a dwell time of 2 hours and finally cooled down to room temperature $\left(1^{\circ} \mathrm{C} \mathrm{min}^{-1}\right)$, yielding the final or brown part.

\subsection{Electrochemical characterization}

To determine the mass transfer properties, the electrodes were housed inside a reactor (Supporting information, Fig. S1). With the electrochemical limiting current technique the mass transfer properties were determined at various flow rates by means of the reversible reaction of potassium ferrocyanide and potassium ferricyanide (Eq. 1).

$$
\left[\mathrm{Fe}(\mathrm{CN})_{6}\right]^{-4} \rightleftharpoons\left[\mathrm{Fe}(\mathrm{CN})_{6}\right]^{-3}+\mathrm{e}^{-}
$$

The flow rate was controlled with a multichannel peristaltic pump (Reglo ICC, Ismatec) and the current response measured at fixed potentials by chronoamperometry. As the flow and concentration profile had to reach steady-state each time the flow rate and potential was varied, linear sweep voltammetry could not be used directly. As an alternative approach, the potential was applied as an incremental "stepwise" function. Each step was applied for 5 minutes, allowing the reactor to reach steady-state both in flow and current response. This steady-state current value was plotted with its corresponding potential to form a discontinuous currentpotential curve, from which the limiting current was determined. Using Eq. 2 the mass transfer coefficient $\left(\mathrm{k}_{\mathrm{L}}\right)$ was calculated from this limiting current value as function of the applied hydrodynamics.

$$
\mathrm{k}_{\mathrm{L}}=\frac{\mathrm{I}_{\text {lim }}}{\mathrm{nFA} \mathrm{Fulk}_{\text {bul }}}
$$

Here $\mathrm{I}_{\text {lim }}$ is the limiting current value, $\mathrm{n}$ the number of transferred electrons in the electrochemical reaction (Eq. 1), $F$ the Faraday constant, $A$ the surface area of the electrode and $C_{\text {bulk }}$ the concentration of ferricyanide in the bulk phase. The catholyte consisted of $25 \mathrm{mM}$ potassium ferricyanide and $0.1 \mathrm{M}$ sodium sulphate as supporting electrolyte. To prevent any limitation effects occurring at the counter electrode the anolyte concentration of potassium ferrocyanide was kept, significantly higher, at $100 \mathrm{mM}$. The sodium sulphate concentration was identical to the catholyte, i.e. $0.1 \mathrm{M}$.

Cyclic voltammetry (CV) was conducted using a solution of $25 \mathrm{mM}$ potassium ferricyanide, $100 \mathrm{mM}$ potassium ferrocyanide and $0.1 \mathrm{M}$ sodium sulphate at a scan rate of $50 \mathrm{mV} / \mathrm{s}$.

\section{Results and Discussion}

\subsection{Electrode fabrication}

The formulation of the paste was optimized with regard to three parameters: (1) mechanical stability of the green part, (2) mechanical stability of the brown part and (3) liquefaction of the paste. Crucial for the mechanical stability of the green part was the amount of epoxy. Below $14.3 \mathrm{wt} \%$ the green part collapsed due to too weak bonds between the nickel particles. However, when the amount of epoxy was raised above $30 \mathrm{wt} \%$ the brown part fell apart. At 30 wt\% too much epoxy was in between the individual nickel particles so when the epoxy was burned off, the nickel particles were too far from one another to uphold the 3D geometry. Consequently the amount of epoxy had to be maintained between $14.3 \mathrm{wt} \%$ and $30 \mathrm{wt} \%$. However, when only epoxy was mixed with nickel particles, the paste jammed, unable to fill the mold. Jamming or bridging typically occurs at solid volume fractions between 0.2-0.6 [19]. However, the solid volume fraction was only 0.17 , but passing a small orifice (e.g. syringe mouth) is reported to increase the solid volume fraction [19] 
Consequently, the viscosity of the paste had to decrease, but without raising the content of epoxy. Therefore a solvent (i.e. glycerol) was added. As the solvent was removed during the mold dissolving step, it did not affect the ratio of epoxy/nickel particles in the green part. Glycerol was chosen above common solvents such water, ethanol and isopropanol. Low viscosity solvents $(<2$ $\mathrm{cP}$ ) still allowed the particles to clog at the syringe mouth. Hence, not only the solid volume fraction is relevant, also the viscosity of the solvent is important. Due to the higher viscosity of glycerol (1412 cP ${ }^{[20]}$ ) the nickel particles could less easily settle, preventing jamming.

Next, the mold had to be removed before sintering, unlike the method of Michorczyk et al. [21]. Burning off the LimoSolve mold material together with epoxy disrupted the electrode geometry as exhaust gasses pushed away the metal particles from one another.

To remove all epoxy and successful sinter the nickel particles, the presence of oxygen was crucial. Under pyrolysis conditions (i.e. argon atmosphere) carbon residuals were still present between the nickel particles even after 48 hours (Fig. 2). Because of the carbon material the nickel particles could not sinter to one another resulting in a mechanical weak brown part. In an oxygen rich atmosphere (i.e. air) all of the epoxy was completely removed. However, due to the presence of oxygen nickel oxidized, which was electrically non-conductive. Consequently nickel oxide was reduced in a second step. Using 5\% hydrogen/argon atmosphere an all-metal 3D electrode mixer was obtained ready for electrochemical testing. Due to the presence of epoxy in the green part, the brown part shrank during sintering. However, limiting the epoxy content to $14.3 \mathrm{wt} \%$, shrinkage of the brown part was not higher than $10 \%$.
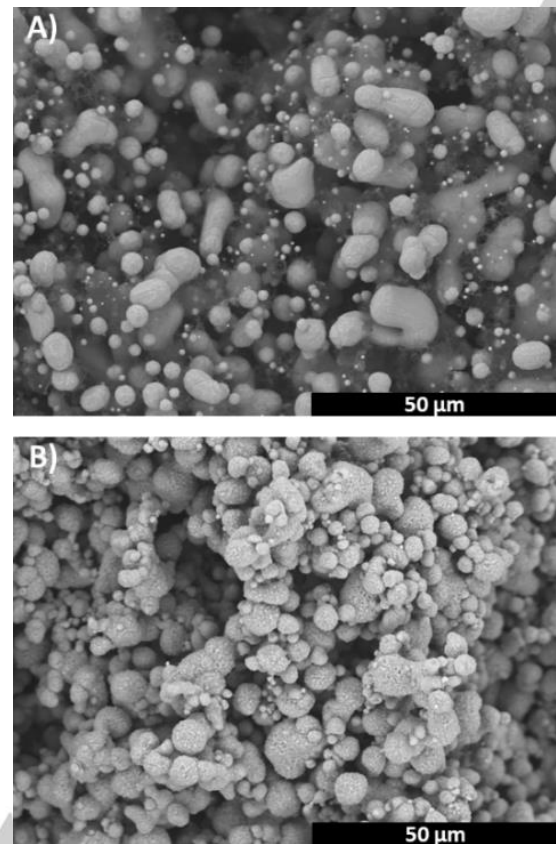

Figure 2. SEM pictures of sintered electrode. A) argon atmosphere B) air atmosphere.
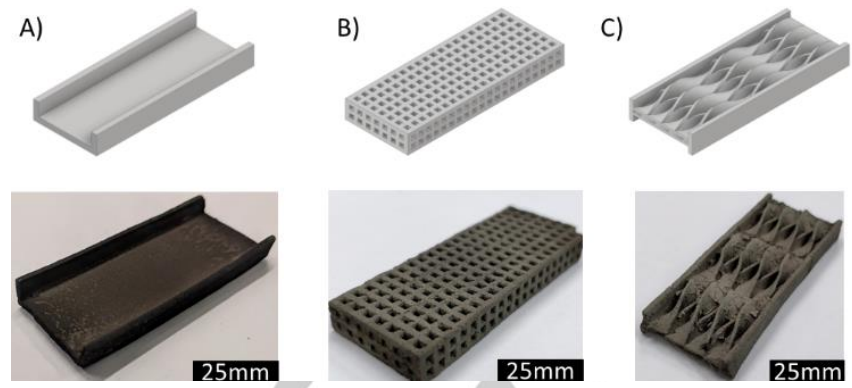

Figure 3. Fabricated electrodes by the indirect 3D printing method: A) flat electrode, B) cubic electrode, C) helical electrode

Three types of electrode designs were fabricated, one flat electrode for benchmarking and two electrode mixers (Fig. 3). The flat electrode had a surface area of $43.2 \mathrm{~cm}^{2}$. The cubic electrode mixer was made of $2 \times 7 \times 19$ cubic unit cells $(3.5 \times 3.5 \times 3.5 \mathrm{~mm})$ and had a surface area of $99.5 \mathrm{~cm}^{2}$. The helical electrode mixer consisted of a row clockwise helixes and a row counterclockwise helixes ${ }^{[22]}$ and had a surface area of $63.0 \mathrm{~cm}^{2}$. Numerical simulations of the proposed electrodes can be found in literature $[6,23,24]$.

\subsection{Electrode characterization}

Prior to the mass transfer characterization measurements the kinetics of the ferri/ferro reaction was studied by CV (Fig. 4) in order to exclude any deactivation of the nickel electrodes, due to the thermal sintering treatment in the fabrication process. From Fig. 4 it was clear that the oxidation and reduction peak still clearly could be observed. Hence, no deactivation occurred during the sintering, making the ferri/ferro reaction suited for the mass transfer characterization.

Using the electrochemical reactor (Fig. S1), the electrode geometries were examined. Two identical electrodes were loaded in the electrochemical reactor, separated by a membrane. The cathode was used as working electrode. From the resulting

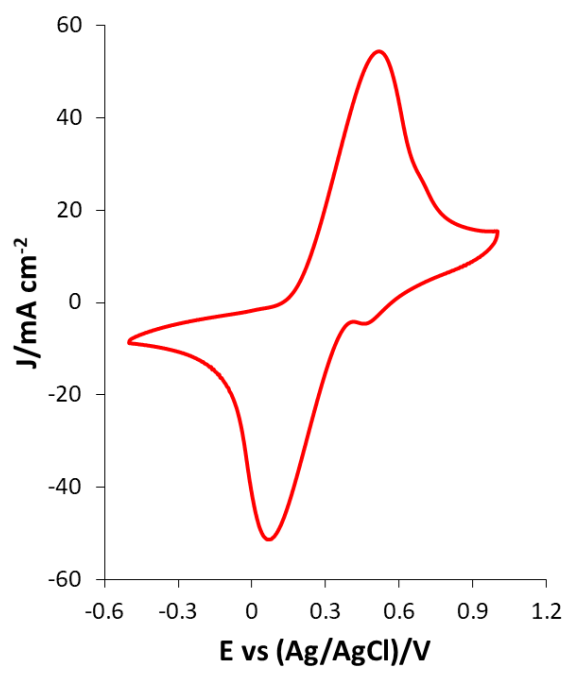

Figure 4. Cyclic voltammogram of sintered nickel at $50 \mathrm{mV} \mathrm{s}^{-1}$, $25 \mathrm{mM} \mathrm{Fe}^{3+}, 100 \mathrm{mM} \mathrm{Fe}^{2+}$ and $0.1 \mathrm{M} \mathrm{Na}_{2} \mathrm{SO}_{4}$. 

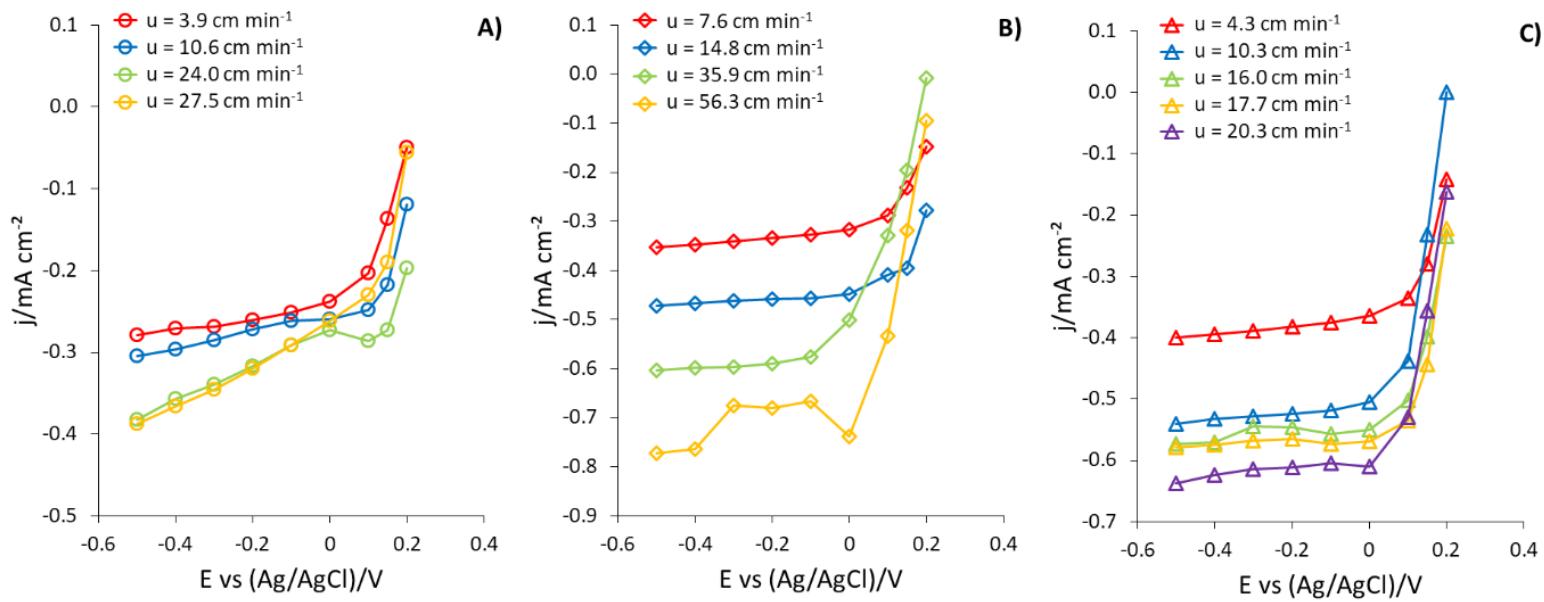

Figure 5. Current-potential response: A) flat electrode, B) cubic electrode, C) helical electrode.

potential-current response (Fig. 5) the limiting current plateau was determined at varying hydrodynamic conditions. To calculate the corresponding mass transfer coefficient (Eq. 2) the limiting current at $-0.3 \mathrm{~V}$ was used for all measured flow rates. From these results (Fig. 6) a similar trend for all electrodes was observed. With increasing velocity (or flow rate) the limiting mass transfer coefficient increased. However, the hydrodynamic effect was much larger for the electrode mixers than for the flat electrode. This could be attributed to the convective mass transfer that was provoked in the electrode mixers. Due to shear forces the boundary layer at the electrode surface was reduced, resulting in higher mass transfer coefficients $[6,25,26]$. As the limiting current plateau only varied slightly, similar values for the mass transfer coefficient were obtained when using the limiting current density at $-0.2 \mathrm{~V}$ or $-0.4 \mathrm{~V}$ (supporting information, Fig. S2). By altering the shape from a conventional flat electrode to the helical electrode the limiting mass transfer coefficient increased up to $47 \%$ at a

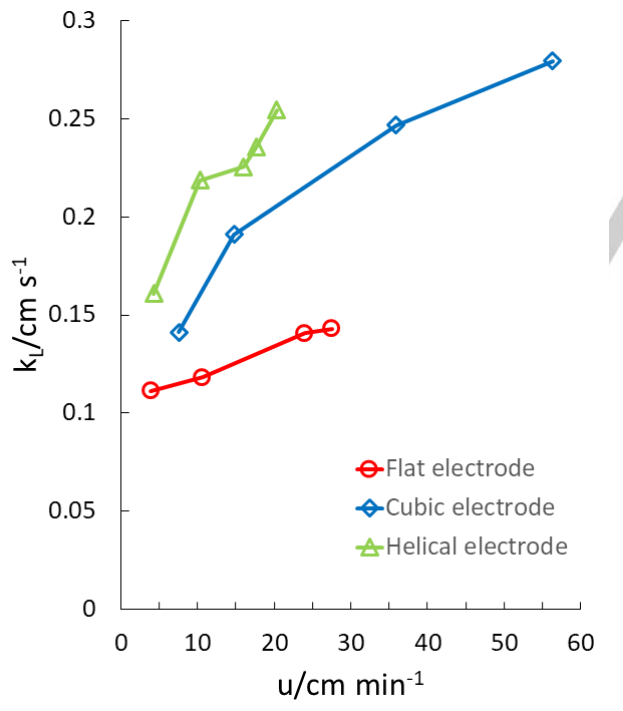

Figure 6. Limiting mass transfer coefficient as a function of the velocity. velocity of $20 \mathrm{~cm} \mathrm{~min}{ }^{-1}$. This demonstrates the valorization potential electrode mixers or 3D electrodes hold to reduce mass transfer limitation and increase productivity or power output of electrochemical reactors or batteries. When comparing the helical electrode with the cubic electrode it is also clear that the helical electrode outperforms the cubic electrode. This is most likely due to less dead zones. It is clear that the electrode design can be optimized, using multiphysics simulations that motivates future research. By demonstrating the potential of indirect 3D printing as fast and cheap alternative for fabricating 3D electrodes with respect to direct 3D printing methods, it allows in combination with CFD simulations to establish a fast prototyping platform to quickly screen various electrode geometries.

\section{Conclusions}

The indirect 3D printing method was presented which allowed to fabricate ordered 3D shaped all-metal electrodes without design restrictions, was easy to implement, robust and had a low cost. Moreover, it only required low-cost $(<€ 10.000)$ off-the-shelf equipment that was easy to operate and did not require high end expertise in advance. Hence, the proposed technique enabled fast-prototyping of all-metal 3D electrode geometries and did not require access to expensive direct 3D printing technology such as SLM. Due to the mixing properties of the structured flow through electrodes, mass transfer was improved up to $47 \%$. Omitting technological high level and high capital cost techniques such as selective laser sintering, further research is motivated to examine the potential of electrode mixers for a variety of electrochemical processes, such as flow batteries or gas evolving reactions, strongly benefiting from the induced shear forces to detach gas bubbles from the electrode surface. 


\section{Acknowledgements}

J. Hereijgers was supported through a postdoctoral fellowship (28761) of the Research Foundation - Flanders (FWO). The authors would like to thank Matteo Adriaens for assisting with the measurements.

Keywords: 3D electrodes e electrode mixer - electrochemistry • electrochemical reactor engineering $\bullet$ additive manufacturing

[1] J. Qi, R. F. Savinell, J. Appl. Electrochem. 1990, 20, 885892.

[2] A. A. Wragg, A. A. Leontaritis, Chem. Eng. J. 1997, 66, 110.

[3] J. L. C. Santos, V. Geraldes, S. Velizarov, J. G. Crespo, Chem. Eng. J. 2010, 157, 379-392.

[4] C. Oduoza, Chem. Eng. J. 2002, 85, 119-126.

[5] A. Ambrosi, J. G. S. Moo, M. Pumera, Adv. Funct. Mater. 2016, 26, 698-703.

[6] J. Lölsberg, O. Starck, S. Stiefel, J. Hereijgers, T. Breugelmans, M. Wessling, ChemElectroChem 2017, 4, 3309-3313.

[7] H. Ragones, S. Menkin, Y. Kamir, A. Gladkikh, T. Mukra, G. Kosa, D. Golodnitsky, Sustain. Energy Fuels 2018, 2, 1542-1549.

[8] Z. Qi, J. Ye, W. Chen, J. Biener, E. B. Duoss, C. M. Spadaccini, M. A. Worsley, C. Zhu, Adv. Mater. Technol. 2018, 3, 1800053.

[9] M. S. Palagonia, D. Brogioli, F. La Mantia, in 69th Annu. ISE Meet., Bologna, 2018.

[10] M. E. Abdelhamid, P. de Souza, T. Rodopoulos, M. Horne, T. Rüther, in 69th Annu. ISE Meet., Bologna, 2018.

[11] D. Golodnitsky, H. Ragones, S. Menkin, Y. Kamir, A. Gladkikh, T. Mukra, G. Kosa, in 69th Annu. ISE Meet., Bologna, 2018.

[12] P. C. Ghimire, A. Bhattarai, R. Schweiss, G. G. Scherer, N. Wai, Q. Yan, Appl. Energy 2018, 230, 974-982.
[13] L. Yu, F. Lin, W. Xiao, L. Xu, J. Xi, Chem. Eng. J. 2019, 356, 622-631.

[14] J. Linkhorst, K. Percin, S. Kriescher, M. Wessling, ChemElectroChem 2017, 4, 2760-2763.

[15] M. Schalenbach, Int. J. Electrochem. Sci. 2018, 11731226.

[16] A. Buttler, H. Spliethoff, Renew. Sustain. Energy Rev. 2018, 82, 2440-2454.

[17] S. M. Saba, M. Müller, M. Robinius, D. Stolten, Int. J. Hydrogen Energy 2018, 43, 1209-1223.

[18] R. Kas, K. K. Hummadi, R. Kortlever, P. de Wit, A. Milbrat, M. W. J. Luiten-Olieman, N. E. Benes, M. T. M. Koper, G. Mul, Nat. Commun. 2016, 7, 10748.

[19] E. Dressaire, A. Sauret, Soft Matter 2017, 13, 37-48.

[20] J. B. Segur, H. E. Oberstar, Ind. Eng. Chem. 1951, 43, 2117-2120.

[21] P. Michorczyk, E. Hędrzak, A. Węgrzyniak, J. Mater. Chem. A 2016, 4, 18753-18756.

[22] C. Fritzmann, M. Hausmann, M. Wiese, M. Wessling, T. Melin, J. Memb. Sci. 2013, 446, 189-200.

[23] G. Yang, B. Weigand, A. Terzis, K. Weishaupt, R. Helmig, Transp. Porous Media 2018, 122, 145-167.

[24] J. Om, W. Wu, P. Ji, in Proc. 2015 Asia-Pacific Energy Equip. Eng. Res. Conf., Atlantis Press, Paris, France, 2015.

[25] H. Schlichting, K. Gersten, Boundary-Layer Theory, Springer Berlin Heidelberg, Berlin, Heidelberg, 2017.

[26] B. Levich, Discuss. Faraday Soc. 1947, 1, 37. 


\section{ARTICLE}

Going 3D: all-metal electrode mixers were fabricated by indirect 3D printing using a robust, easy and cheap method. Utilizing the third dimension the limiting mass transfer coefficient was increased by $47 \%$.

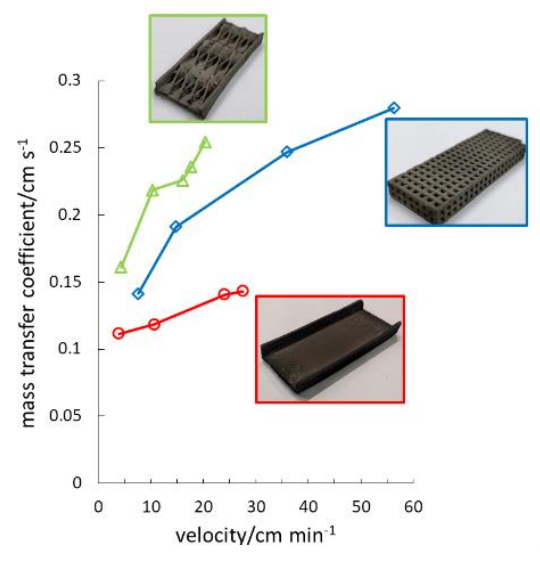

Jonas Hereijgers, Jonathan Schalck, Jonas Lölsberg, Matthias Wessling, Tom Breugelmans

Page No. - Page No.

Indirect 3D printed electrode mixers 